\title{
Eine philologische Note zum Wort „Hmk.t“ in pChester Beatty VII, vso I, 5
}

\author{
Stefan Bojowald \\ Universität Bonn
}

\begin{abstract}
In this article, the word $=-1\}=$ from pChester Beatty VII, vso I, 5 is examined again. The determinative has indicated only the denotation of a water body, and previous attempts of interpretation could not convince. Here, a connection with "HmAg" "surround" is assumed. The word is determined as the name of a waterscape surrounded by trees and shrubs.
\end{abstract}

Keywords: Egyptian philology; new interpretation for pChester Beatty VII, vso I, 5; new explanation for $-4 \mathrm{~N} U 1 \equiv \bar{\square}$.

Im Zentrum dieses Beitrags wird eine Passage aus dem ägyptischen pChester Beatty VII, vso I, 5 stehen, der nach paläographischen Kriterien in die Zeit von Ramses II. datiert werden kann. Der Papyrusabschnitt setzt mit einer Ritualanweisung ein, auf die eine längere Lücke folgt. Die heute erhaltenen Textreste erlauben aber die Rekonstruktion einer mythologischen Episode aus dem Leben der Götter Seth und Anat, in welcher der Same/das Gift in Gestalt einer schönen Frau ein kühles Bad genommen hat. Die Anekdote ist in den 
Worten ,Sn.t n.t si ... c m pA xApw Hr wab=c m pA“ erzählt worden, die in der Erstübersetzung von Gardiner mit „Incantation for a man ... [The goddess Anat was disporting ?] herself in the (stream of) $\mathrm{Khap}^{2}$ and bathing in the (stream of) Hemket ${ }^{\text {" } 3}$ ins Englische übertragen worden sind. Die Geschichte ist anschließend noch weitergegangen, was hier aber vorerst nicht $\mathrm{zu}$ interessieren braucht. Die jüngeren Übersetzungen haben eine andere Aufteilung gewählt und nach der Lücke einen neuen Satz beginnen lassen. Im Ergebnis hat dieser Schritt zu Übersetzungen wie „She was at the shore, bathing in the Hmkt ${ }^{* 4}$ und ,She was on the $\mathrm{x}$. bathing herself in the Hamakata" 5 geführt. In der Zwischenzeit waren aber für den Anfang Parallelen ${ }^{6}$ bekannt geworden, welche die Ergänzung ,tA mtw.t Hr wab m pA xpw“ „Der Same/ das Gift badete sich in xpw“ wahrscheinlich machen. Der hier vorgelegte Beitrag wird sich intensiver mit dem Wort $= \pm 10=\frac{1}{\square}$ beschäftigen, das trotz mehrfacher Anläufe noch nicht zufriedenstellend bestimmt ist.

Die Lage wird dadurch noch zusätzlich erschwert, dass das Wort offenbar nur an dieser einen Stelle belegt ist. Das Determinativ der „Liegenden Wasserlinien“ kommt hierbei zunächst nicht zur Hilfe, da es lediglich auf die Bezeichnung für ein stehendes oder fließendes Gewässer hindeutet. Die Wörter

\footnotetext{
${ }^{1}$ Gardiner 1935 (Plates), Pl. 36 vso 1, 5.

${ }^{2}$ Die Bedeutung des Wortes „xApw“ kann im Licht der neueren Forschung exakter eingegrenzt werden: zur Bedeutung „Ufer“ vgl. Helck 1971, 518; van Dijk 1986, 34; Schneider 2003, 620; zu einem Zusammenhang des Wortes mit luwisch „xäpa/ï-“ „Fluß“ vgl. Schneider 2004, 24; Breyer 2004, 267; dagegen Simon 2010, 81, der wieder der Bedeutung „Ufer“ zuneigt.

${ }^{3}$ Gardiner 1935 (Text), 62.

${ }^{4}$ Hoch 1994, 227.

${ }^{5}$ Simon 2010, 81 .

${ }^{6}$ van Dijk 1986, 32-33.

${ }^{7}$ Wreszinski 1912, $150(10,6)$. Die ägyptische „Hmk.t“ - Krankheit ist von Ebbell 1924, 149, mit der arabischen Krankheitsbezeichnung D DÖb in Verbindung gebracht worden. In der medizinischen Frage dieser Krankheit hat er eventuell an Scharlach gedacht. In dieser Hinsicht sind von Deines/Westendorf 1962, 603, allerdings zu keiner evidenten Lösung gelangt. Die Krankheit ist von Steiner 1992, 198, mit ugaritisch „, ilt xnqt" "strangling goddess" und „Hnq.t" "strangulation - demon" aus Arslan Tasch gleichgesetzt worden. Die Position ist auch von Schneider 1996, 176, befürwortet worden, der den von ihm selbst aufgestellten Zusammenhang mit hethitisch „xenkan/xinkan“ „Seuche/Pest“ für problematischer hält. Der Vorschlag von Steiner ist auch bei Simon 2010, 83, ausdrücklich gebilligt worden. Die Vorgaben
} 
London 10,6 und $=\mathbb{N}$ e 8 aus p. Med. London 11, 7 weisen zwar in der Lautstruktur zunächst keine größeren Unterschiede auf, stellen sich aber bei näherer Betrachtung als Schreibungen für die „Hmk.t Krankheit heraus. Das Determinativ des sitzenden Mannes könnte im letzten Fall ein Indiz für die Vorstellung der Krankheit als Dämon bilden $^{9}$. Das gleiche Wort ist in p. Brit. Mus. 10083, vso. $42-43$; p. Turin Museum 1984, rto. 106 und in p. Turin Museum 1983 , vso 30/31 1640 recto $\mathrm{x}+3^{11}$ ist außerdem ein Wort 1000 in alphabetischer Schreibung attestiert, das von Mathieu ${ }^{12}$ mit derselben Krankheit in Zusammenhang gebracht worden ist.

Die folgenden Zeilen versuchen auf möglichst innovative Weise hinter die Bedeutung von $\angle A D Q=\square$ zu schauen. Der hier gemachte Vorschlag zeichnet sich gegenüber allen bisherigen Versuchen dadurch aus, dass er von außen durch eine Parallele gestützt werden kann. Die eigenen Argumente sollen nach dem obligatorischen Forschungsüberblick präsentiert werden.

In der Editio princeps des Textes hatte Gardiner ${ }^{13}$ noch von einer Übersetzung des Wortes abgesehen und sich mit der reinen Wiedergabe in englischer Transkription, scil. „Hemket“, begnügt (s. o.).

Das Wort war danach von Albright ${ }^{14}$ mit dem ugaritischen Begriff „Smk“ aus KTU 1. 10, II, 9. 12 in Verbindung gebracht worden, der eine sumpfige Gegend bezeichnet, in der sich Baal und Anat ein Stelldichein gegeben haben. Der Vorschlag hat jedoch bei van Dijk ${ }^{15}$ Kritik geerntet, der auf linguistische Ungereimtheiten und

von Steiner sind bei Fischer - Elfert 2005, 35, zur Bestimmung der Krankheit als „Scharlachfieber“ genutzt worden. Die Identifikation der ägyptischen „Hmk.t“ Krankheit mit proto-semitisch „*xanqatu“ „strangulation“ ist hingegen von Leitz 1999, 61 n. 96, aus lautlichen Gründen abgelehnt worden.

${ }^{8}$ Wreszinski 1912, $152(11,7)$.

${ }^{9}$ Steiner 1992, 198 .

${ }^{10}$ Edwards 1960,11 n. 28/58 n. 31/66 n. 63.

${ }^{11}$ Posener 1980, 90/Pl. 66-66a.

${ }^{12}$ Mathieu 2008, 65b.

${ }^{13}$ Gardiner 1935 (Text), 62 n. 6.

${ }^{14}$ Albright 1946, 197 n. 39.

${ }^{15}$ van Dijk 1986, 34. 
fehlende Gemeinsamkeiten der ägyptischen Geschichte mit dem ugaritischen Mythos verwiesen hat.

In späterer Zeit hat Helck ${ }^{16}$ das Wort in seine Liste semitischer Fremdwörter aufgenommen, wo es die Bedeutung „Bach“ erhalten hat. Das Wort kennt er nur aus Chester Beatty VII vso 1,5 , ohne es etymologisch erklären zu können. In der Neuauflage ${ }^{17}$ seiner Arbeit sind diese Koordinaten unverändert übernommen worden.

Der nächste Kommentar zu diesem Wort ist von Hoch ${ }^{18}$ geschrieben worden, der probeweise die Bedeutung „body of water“ vorschlägt. Das Wort hat er als Bildung mit „m“ - Präfix identifiziert, was zusätzlich die Annahme einer Metathese erforderlich macht. In Bezug auf die Etymologie des Wortes tritt er vorsichtig für einen Zusammenhang mit den hebräischen Wurzeln עמקים tiefes Wasser“ oder עמקות, tiefe Dinge“ ein. Die Gleichung bereitet auf lautlicher Ebene keine Schwierigkeiten, da alle nötigen Regeln eingehalten werden. Die Ausführungen von Hoch lassen jedoch erkennen, dass er nicht mit letzter Überzeugung hinter seinem eigenen Vorschlag gestanden hat. Das maskuline Demonstrativpronomen „pA“ vor $=\wedge .10=$ schließt nach seiner Meinung ein Femininum aus, weshalb die Gruppe 14 Wurzelkonsonanten bezeichnen müsse. Im unteren Teil wird die Sprache noch einmal auf dieses Thema kommen, wo auch eigene Ursachenforschung zu dieser Frage betrieben wird.

Die Reihe der bisherigen Interpreten ist von Schneider ${ }^{19}$

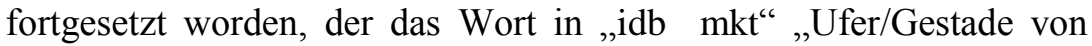
Megiddo (?)“ emendieren wollte. Die These hatte er mit dem Hinweis auf die große Ähnlichkeit von $=$ und ${ }^{\rightleftarrows}$ im Hieratischen erläutert. Die Zeichen " wären demnach als logographische Schreibung für „idb“ aufzufassen. In der Ähnlichkeit der beiden hieratischen Zeichengruppen muss Schneider zwar zweifellos Recht gegeben werden. Der Vorschlag stimmt aber dennoch bedenklich, weil keine weiteren Belege für die Wortverbindung aus „idb“ „Ufer/Gestade“ und „mkt" „Megiddo“ genannt worden sind. In der Tat dürften sie schwer zu finden sein. Die Schreibung für Megiddo scheint auch nicht ganz dem herkömmlichen Standard zu entsprechen. Die Verlegung

\footnotetext{
${ }^{16}$ Helck 1962, 566.

${ }^{17}$ Helck 1971, 518.

${ }^{18}$ Hoch 1994, 227.

${ }^{19}$ Schneider 2003, 620 .
} 
des Schauplatzes der Ereignisse ausgerechnet nach Megiddo stellt ohnehin eine reine Vermutung dar. Das in Klammern gesetzte Fragezeichen nach Megiddo deutet denn auch auf die eigene Skepsis von Schneider hin. Der Ansatz hat obendrein gegen die goldene Regel verstoßen, dass Textein-griffe - egal in welcher Form - immer nur äußerstes Mittel sein sollten.

In diesem Beitrag wird daher eine etwas andere Herangehensweise gewählt. Die Emendation von Schneider wird sich dabei als unnötig herausstellen. Die Notwendigkeit zur Ansetzung eines semitischen Fremdwortes wird ebenfalls entfallen. Die semitische Wurzelfrage hatte Hoch ohnehin vor eine ernste Herausforderung gestellt. Das Resultat der folgenden Überlegungen wird hoffentlich zeigen, dass das Problem viel einfacher zu bewältigen ist. In diesem Kontext braucht nur das Stichwort genannt werden, dass auch ägyptische Wörter in Syllabischer Schrift geschrieben werden konnten. In der Vergangenheit ist bereits auf mehrere Fälle dieser Art aufmerksam gemacht worden ${ }^{20}$. Das Wort $=\mathbb{A} 01=\bar{E}$ kann jetzt diesem Corpus hinzugefügt werden.

Der genuin ägyptische Charakter von $=-101=\pi$ fordert zur Suche nach einer geeigneten Wurzel heraus. Das Wort soll dementsprechend von der Wurzel „HmAg“ abgeleitet werden, die in den Wörterbüchern mit der Bedeutung ,umschließen“'21 $\mathrm{zu}$ finden ist. In besonderem Maße kommt es hierbei auf den bildlichen Gebrauch

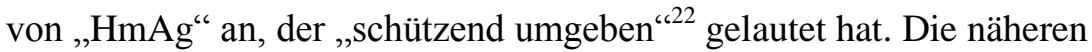
Einzelheiten werden im nächsten Abschnitt stärker akzentuiert. Das

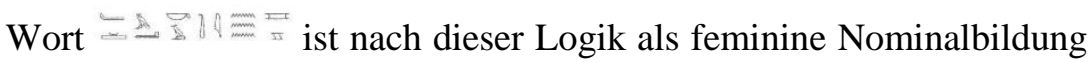
von der verbalen Wurzel „HmAg“ zu betrachten. Die hierfür notwendigen phonetischen Voraussetzungen sind leicht einzuhalten, da lediglich der Lautwandel zwischen , $\mathrm{g}^{6}$ und , $\mathrm{K}^{623}$ sowie der Ausfall

\footnotetext{
${ }^{20}$ Smither 1941, 131; Fecht 1960, 85; Quack 1994, 55; Fischer-Elfert 1986 (Satirische Streitschrift), 161/168; Fischer-Elfert 1986 (Ostraka), 76; Fischer-Elfert 1997, 22/49.

${ }^{21}$ WB III, 94, 8; Takács 1999, 153.

${ }^{22}$ WB III, 94,9 .

${ }^{23}$ Westendorf 1962, 43. Der Lautwandel ist zwar von Westendorf als unsicher markiert worden, in der Spätzeit kommt er jedoch öfter vor, vgl. z. B. Jasnow/Zauzich 2005, 89. In der Schreibung „,bg“ für den „,bik“ - Falken bei Osing 1998, 245c, ist er überdies eindeutig nachgewiesen. Die Synkope von „¡“ soll an anderer Stelle abgehandelt werden.
} 
von „A“ ${ }^{\text {‘24 }}$ zu erfolgen hat. In diesem speziellen Fall kann sogar darauf verwiesen werden, dass in der Spätzeit für „HmAg“ die Schreibung

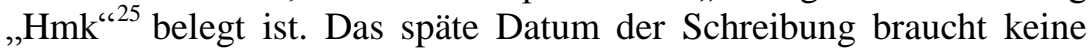
Zweifel an der Vertretbarkeit der gesamten These hervorzurufen. Die Wahrscheinlichkeit der Deutung lässt sich vielmehr dadurch noch um einige Prozente erhöhen, dass in der Schreibung $= \pm \Delta \mathbb{\mathbb { A }} i^{26}$ für den „HmAg.t ${ }^{627}$ - Stein eine fast identische Erscheinung aus dem Neuen Reich zu beobachten ist. Die Nähe zwischen $=\Delta \mathbb{A} i$ und dem „HmAg.t" - Stein ist auch von Caminos ${ }^{28}$ behauptet worden. Die Schreibung stellt eine höchst willkommene Parallele aus der gleichen Zeitstufe wie die Ableitung des Wortes $=50$ von der Wurzel „HmAg“ „umschließen“ dar. Die unterschiedliche Wiedergabe des Gutturals sollte nicht überbewertet werden, da die Grenzen zwischen „k“ und „," durchlässig waren ${ }^{29}$. Das Wort $=\Delta \mathbb{\mathbb { N }}$ ist sinniger Weise ebenfalls syllabisch geschrieben worden. Das Wort „HmAg“ „umschließen“ ist mit der Angabe „spät/griechisch“ ins Wörterbuch gesetzt worden. Wenn die hier vorgelegten Bemerkungen aber stimmen sollten, ist das Wort mindestens ein halbes Jahrtausend älter gewesen.

Was die inhaltliche Seite betrifft, so ergibt sich die Schlussfolgerung, dass das Wort einen Teich oder Wasserlauf bezeichnet hat, deren Uferränder vielleicht mit Bäumen oder Sträu-

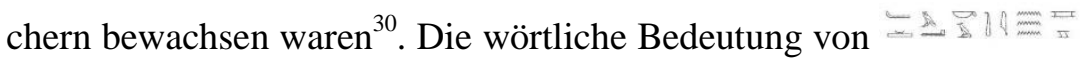
kann demnach mit „die Umgebene (scil. Wasserfläche)“ umschrieben werden. Die dichte Vegetation hat dabei einen Sichtschutz geboten, welcher die weibliche Personifikation des Samens/Giftes vor den Blicken neugieriger Zuschauer abgeschirmt hat. Der durch die Sonderbedeutung von „HmAg“ intendierte Schutzaspekt kann vor

\footnotetext{
${ }^{24}$ Westendorf 1962, 9 .

${ }^{25}$ Leitz 2010, 328.

${ }^{26}$ WB III, 99, 10.

${ }^{27}$ Harris 1961, 118; Lesko 1984, 113; Meeks 1980, 247; Meeks 1981, 249-250; Aufrère 1991 (Vol. I), 180/ 217/250/285/339; Aufrère 1991 (Vol. II), 375/557558/757/783/819; Hannig 2006, 1685

${ }^{28}$ Caminos 1954, 442. Der „Hmq”- Edelstein ist hingegen von Burchardt 1910, II 35, unter Verweis auf den „Hqm“ - Edelstein als eigenes Wort analysiert worden.

${ }^{29}$ Westendorf 1962, 43.

${ }^{30}$ Zur Vorstellung von Bäumen als Umrandung von Wasserflächen vgl. Youotte 1982-1983, 130-132; Guermuer 2005, 132-133; Leitz 2011, 369.
} 
diesem Hintergrund als erfüllt gelten. Die Dame hat auf diese Weise für die Körperpflege einen intimen Ort gefunden, an dem sie sich ganz der Reinigung hingeben konnte. Der weitere Verlauf der Handlung zeigt zwar, dass die Badende dennoch von Seth erspäht wird, woraufhin es zum sexuellen Kontakt kommt. Die Tatsache würde aber an der hiesigen Interpretation erst einmal nichts ändern, da die Dinge immer eine überraschende Wende nehmen können. Der Geschlechtsverkehr scheint ohnehin nicht in gegenseitigem Einverständnis stattgefunden zu haben ${ }^{31}$.

Im Schlussteil soll noch einmal zum Verhältnis zwischen dem maskulinen Artikel „pA“ und Die oben geschilderte Idee von Hoch war nicht so zwingend, dass sie keine andere Erklärung neben sich duldet. Die Stimme soll hier daher zu einer Gegenmeinung erhoben werden. In diesem Sinn lässt sich sagen, dass die Annahme eines simplen Versehens des ägyptischen Schreibers den Kern der Sache wesentlich besser treffen dürfte. Der Irrtum könnte sehr leicht damit entschuldigt werden, dass der Schreiber oder Kopist nicht mehr/noch nicht richtig über das Genus des syllabisch geschriebenen Wortes informiert war.

\section{Bibliographie}

Albright, William Foxwell. Archaeology and the Religion of Israel, (Baltimore 1946²).

Aufrère, Sydney. L'univers minéral dans la pensée Égyptienne, 1. L'influence du désert et des minéraux sur la mentalité des anciens Égyptiens, Bibliothèque d' Étude, t. CV/I (Le Caire: Institut Francais d' Archéologie Orientale du Caire, 1991).

Aufrère, Sydney. L' univers minérals dans la pensée Égyptienne, Volume 2, Les minerais, les métaux, les minéraux et les produits chimiques, les trésors et les défilés de contrées minières: leur intéfration dans la marche de l' univers et l' entretien de la vie divine, Vol II, Bibliothèque d'Étude t. CV/2 (Le Caire: Institut Francais d' Archéologie Orientale du Caire, 1991).

\footnotetext{
${ }^{31}$ Stadelmann 1967, 132-133; dagegen: van Dijk 1986, 35/44.
} 
Breyer, Francis Amadeus Karl. Anatolisches Sprachmaterial in ägyptisch - hieroglyphischen Inschriften, ein Vorbericht, in: Thomas Schneider, Das Ägyptische und die Sprachen Vorderasiens, Nordafrikas und der Ägäis, Akten des Basler Kolloquiums zum ägyptisch - nichtsemitischen Sprachkontakt Basel 9. - 11. Juli 2003, Alter Orient und Altes Testament 310 (Münster: Ugarit-Verlag, 2004), 259-270.

Burchardt, Max. Die altkanaanäischen Fremdworte und Eigennamen im Aegyptischen, Zweiter Teil, Listen der syllabisch geschriebenen Worte sowie der altkanaanäischen Fremdworte und Eigennamen (Leipzig: J. C. Hinrichs'sche Buchhandlung, 1910).

Caminos, Ricardo A. Late - Egyptian Miscellanies, Brown Egyptological Studies I (Oxford: University Press, 1954).

von Deines, H./Westendorf, W. Wörterbuch der medizinischen Texte, Zweite Hälfte $(\mathrm{h}-\mathrm{D})$, Grundriss der Medizin der alten Ägypter VII 2 (Berlin: Akademie - Verlag, 1962).

van Dijk, Jacobus. aAnat, Seth and the Seed of Prëa, in: H. L. J. Vanstiphout/K. Jongeling/F. Leemhuis/J. G. Reinink, Scripta Signa Vocis, Studies about Scripts, Scriptures, Scribes and Languages in the Near East, presented to J. H. Hospers by his pupils, colleagues and friends (Groningen: Egbert Forsten, 1986).

Ebbell, Bendix. Die ägyptischen Krankheitsnamen, Zeitschrift für ägyptische Sprache und Altertumskunde 59 (1924), 144-149.

Edwards, Iorwerth Eiddon St. Hieratic Papyri in the British Museum, Fourth Series, Oracular Amuletic Decrees of the Late New Kingdom, Vol. I, Text (London: The Trustees of the British Museum, 1960).

Fecht, Gerhard. Amarna-Probleme (1-2), Zeitschrift für Ägyptische Sprache Altertumskunde 85 (1960), 83-118.

Fischer-Elfert, Hans-Werner. Die Satirische Streitschrift des Papyrus Anastasi I, Übersetzung und Kommentar, Ägyptologische Abhandlungen 44 (Wiesbaden: Harrassowitz, 1986). 
Fischer-Elfert, Hans-Werner. Literarische Ostraka der Ramessidenzeit in Übersetzung, Kleine Ägyptische Texte 9 (Wiesbaden: Harrassowitz, 1986).

Fischer-Elfert, Hans- Werner. Lesefunde im literarischen Steinbruch von Deir el-Medineh, Kleine Ägyptische Texte 12 (Wiesbaden: Harrassowitz, 1997).

Fischer-Elfert, Hans-Werner. Abseits von Ma'at, Fallstudien zu Außenseitern im Alten Ägypten,Wahrnehmungen und Spuren Altägyptens, Kulturgeschichtliche Beiträge zur Ägyptologie Band 1 (Würzburg, 2005).

Gardiner, Alan H. Hieratic Papyri in the British Museum, Third Series, Chester Beatty Gift, Vol. I, Text (London: British Museum, 1935).

Gardiner, Alan H. Hieratic Papyri in the British Museum, Third Series, Chester Beatty Gift, Vol. II, Plates (London: British Museum, 1935)

Guermeur, Ivan. Les Cultes d' Amon hors de Thèbes, Recherches de Géographie religieux, Bibliothèque de l' École des Hautes Études Sciences Religieuses, Volume 123 (Turnhout: Brepols, 2005).

Hannig, Rainer. Ägyptisches Wörterbuch II, Mittleres Reich und Zweite Zwischenzeit (Mainz: von Zabern, 2006).

Harris, James R. Lexicographical Studies in Ancient Egyptian Minerals, Deutsche Akademie der Wissenschaften zu Berlin, Insti-tut für Orientforschung, Veröffentlichung Nr. 54 (Berlin: Akademie - Verlag, 1961).

Helck, Wolfgang. Die Beziehungen Ägyptens zu Vorderasien im 3. und 2. Jahrtausend v. Chr. Ägyptologische Abhandlungen 5 (Wiesbaden: Harrassowitz, 1962).

Helck, Wolfgang. Die Beziehungen Ägyptens zu Vorderasien im 3. und 2. Jahrtausend v. Chr., 2. verbesserte Auflage, Ägyptologische Abhandlungen 5 (Wiesbaden: Harrassowitz, 1971). 
Hoch, James E. Semitic words in Egyptian texts of the New Kingdom and Third Intermediate Period (Princeton: Princeton University Press, 1994).

Jasnow, R./Zauzich, K. - Th. The ancient Egyptian Book of Thot, A Demotic Discourse on Knowledge and Pendant to the Classical Hermetica, Volume 1: Text (Wiesbaden: Harrassowitz Verlag, 2005).

Leitz, Christian. Hieratic Papyri in the British Museum VII, Magical and Medical Papyri of the New Kingdom (London: The Trustees of the British Museum, 1999).

Leitz, Christian. Der Lobpreis des Krokodils, Drei Sobekhymnen aus Kom Ombo, in: Hermann Knuf/Christian Leitz/Daniel von Recklinghausen, Honi Soit Qui Mal Y Pense, Studien zum pharaonischen, griechisch - römischen und spätantiken Ägypten zu Ehren von Heinz - Josef Thissen, Orientalia Lovaniensia Analecta 194 (Leuven - Paris - Walpole, MA: Uitgeverig Peeters/Departement Oosterse Studies, 2010), 291355.

Leitz, Christian. Der Sarg des Panehemesis in Wien, Studien zur Spätägyptischen Religion 3 (Wiesbaden: Harrassowitz, 2011).

Mathieu, Bernard. Cuisine sans set une interpretation de 1' ostracon magique O. DeM 1640, Göttinger Miszellen 218 (2008), 63-70.

Meeks, Dimitri. Annee Lexicographique, Tome I (1977) (Paris, 1980).

Meeks, Dimitri. Annee Lexicographique, Egypte Ancienne, Tome 2 (1978) (Paris, 1981).

Osing, Jürgen. Hieratische Papyri aus Tebtunis I, Text, The Carlsberg Papyri 2, CNI Publications 17 (Kopenhagen: Museum Tusculanum Press, 1998).

Quack, Joachim Friedrich. Die Lehren des Ani, Ein neuägyptischer Weisheitstext in seinem kulturellen Umfeld, Orbis Biblicus et Orientalis 141 (Freiburg Schweiz: Universitätsverlag/ Göttingen: Vandenhoeck \& Ruprecht, 1994).

Schneider, Thomas. Rezension zu: James E. Hoch, Semitic Words in Egyptian Texts of the New Kingdom and Third Intermediate 
Period: Princeton (New Jersey), Princeton University Press, 1994, XXI - 572 p. 15,8 x 24, 2, \$65, 00/50, 00, Orientalia 65 (1996), 174-177.

Schneider, Thomas. Texte über den syrischen Wettergott aus Ägypten, Ugarit - Forschungen 35 (2003).

Schneider, Thomas. Nichtsemitische Lehnwörter im Ägyptischen. Umriß eines Forschungsgebietes, in: Thomas Schneider, Das Ägyptische und die Sprachen Vorderasiens, Nordafrikas und der Ägäis, Akten des Basler Kolloquiums zum ägyptisch nichtsemitischen Sprachkontakt Basel 9. - 11. Juli 2003, Alter Orient und Altes Testament 310 (Münster: Ugarit-Verlag, 2004), 11-31.

Simon, Zsolt. Hethitisch - luwische Fremdwörter im Ägyptischen?, Göttinger Miszellen 227 (2010), 77-92.

Smither, Paul. A Ramesside Love Charm, The Journal of Egyptian Archaeology 27 (1941), 131-132.

Stadelmann, Rainer. Syrisch - Palästinensische Gottheiten in Ägypten, Probleme der Ägyptologie, Fünfter Band (Leiden: Brill, 1967).

Steiner, Richard C. Northwest Semitic Incantations in an Egyptian Medical Papyrus of the Fourteenth Century B. C. E., Journal of Near Eastern Studies 51 (1992), 191-200.

Takács, Gábor. Etymological Dictionary of Egyptian, Volume One: A Phonological Introduction, Handbuch der Orientalistik, Erste Abteilung, Der Nahe und Mittlere Osten, Achtundvierzigster Band (Leiden/Boston/Köln: Brill, 1999).

Ward, William A. Notes on Egyptian Group-Writing, Journal of Near Eastern Studies 16 (1957), 198-203.

Westendorf, Wolfhart. Grammatik der medizinischen Texte, Grundriss der Medizin der alten Ägypter VIII (Berlin: Akademie - Verlag, 1962).

Wreszinski, Walter. Der Londoner Medizinische Papyrus (Brit. Museum Nr. 100059) und der Papyrus Hearst in Transkription, 
Übersetzung und Kommentar (Leipzig: J. C. Hinrichs'sche Buchhandlung, 1912).

Yoyotte, Jean. L'Amon de Naukratis, Reveu d' Égyptologie 34 (1982-1983), 129-136. 